\title{
Original
}

\section{Effect of Low-Intensity Pulsed Ultrasound on Bone-Healing Process in Murine Low-Turnover Osteoporosis Model}

\author{
Aritomo Yoshida ${ }^{1,2)}$, Hodaka Sasaki ${ }^{1,2)}$, Yoshitaka Furuya ${ }^{1,2)}$, Masao Yoshinari ${ }^{2)}$ and Yasutomo Yajima ${ }^{1,2)}$ \\ 1) Department of Oral and Maxillofacial Implantology, Tokyo Dental College, Chiba, Japan \\ 2) Division of Oral Implants Research, Oral Health Science Center, Tokyo Dental College, Chiba, Japan
}

(Accepted for publication, April 9, 2013)

\begin{abstract}
The effects of postmenopausal osteoporosis for bone defect healing processes have already been reported, but not for senile osteoporosis caused by low-turnover metabolism. Low-Intensity-Pulsed Ultrasound (LIPUS) is known to promote bone defect healing in high-turnover osteoporosis an animal model. The aim of this study was to investigate the effect of LIPUS on the bone-healing process in a low-turnover osteoporosis model using the Senescence-Accelerated Mouse Prone 6 (SAMP6) strain of mice. Twenty-week-old SAMP6 and Senescence-Accelerated Mouse Resistant (SAMR1) mice were used as senile osteoporosis and normal aging models, respectively. Bone defects (diameter, $0.9 \mathrm{~mm}$ ) were created in the both SAMP6 and SAMR1 femurs. At 7days after surgery, the LIPUS irradiation groups of SAMR1 (R1LG) and SAMP6 (P6LG) were exposed to LIPUS (1.0 MHz, $320 \mathrm{~mW}, 15 \mathrm{~min} /$ day) for 6 days. The non-irradiation groups of SAMR1 (R1CG) and SAMP6 (P6CG) were used as controls. All groups were sacrificed at 14 days after creation of bone defects. Radiological analysis, histological evaluation and immunohistochemical staining for osteocalcin (OC) were performed. From the radiological evaluation, the new bone of defected area in SAMR1 group showed cortical bone-like structure, but that in the SAMP6 group showed trabecular bone-like structures. The increase in bone area in P6LG was greater than that in P6CG according to chronological change analysis using X-ray micro-CT $(\mathrm{p}<0.01)$. Histological analysis revealed outward new bone formation originating in the periosteum in P6LG. Positive reaction for $\mathrm{OC}$ was localized on the surface of new bone in P6CG, whereas that in P6LG was observed over the whole region of new bone, from the outer to the bone marrow side.

These results showed that LIPUS accelerates healing on low-turnover osteoporosis by promoting bone formation from periosteum and supplementing reduced bone formation from bone marrow.
\end{abstract}

Key words: Dental implant, Bone healing, Senile osteoporosis, Low-intensity pulsed ultrasound

\section{Introduction}

Systemic disease is a significant problem in dental implant treatment. Osteoporosis, a metabolic bone disease accompanying old age, has been reported as a risk factor in implant treatment, as the success of implant treatment is defined as achieving and maintaining osseointegration ${ }^{1,2)}$. In post-menopausal osteoporosis, bone resorption increases due to estrogen deficiency; in senile osteoporosis, ossification potential is compromised due to aging ${ }^{3)}$. Postmenopausal osteoporosis is a high-turnover osteoporosis resulting in trabecular bone reduction, while senile osteoporosis is a low-turnover osteoporosis affecting both cortical and trabecular bone ${ }^{4}$.

Many studies have utilized ovariectomized (OVX) animal

Correspondence to: Dr. Hodaka Sakaki, Department of Oral and Maxillofacial Implantology, Tokyo Dental College, 1-2-2 Masago, Mihamaku, Chiba, 261-8502 Japan; Phone: +81-43-270-3573; Fax: +81-43-2703574; E-mail: hosasaki@tdc.ac.jp models for investigation of postmenopausal osteoporosis. Some have reported a reduction in trabecular bone and delay of fracture or bone defects ${ }^{5,6)}$, while others a reduction in bone-to-implant contact ratio $^{7)}$. On the other hand, studies on senile osteoporosis have used the Senescence-Accelerated Mouse Prone 6 (SAMP6) strain of mice, a type that develops low-turnover osteoporosis ${ }^{8}$. These studies have reported a decline in thickness of trabecular and cortical bone, bone mineral density (BMD) and femur weight due to osteoblastic hypoplasia ${ }^{9,10)}$. However, to our knowledge, no studies to date have investigated the effect of low-turnover osteoporosis on implant therapy or the healing process in bone defects.

Ultrasound is a form of energy that is transmitted through biological tissues as high-frequency acoustic waves, and is widely used in medicine as a diagnostic, therapeutic, and operative tool ${ }^{11}$. Low-intensity pulsed ultrasound (LIPUS) with frequency intervals 


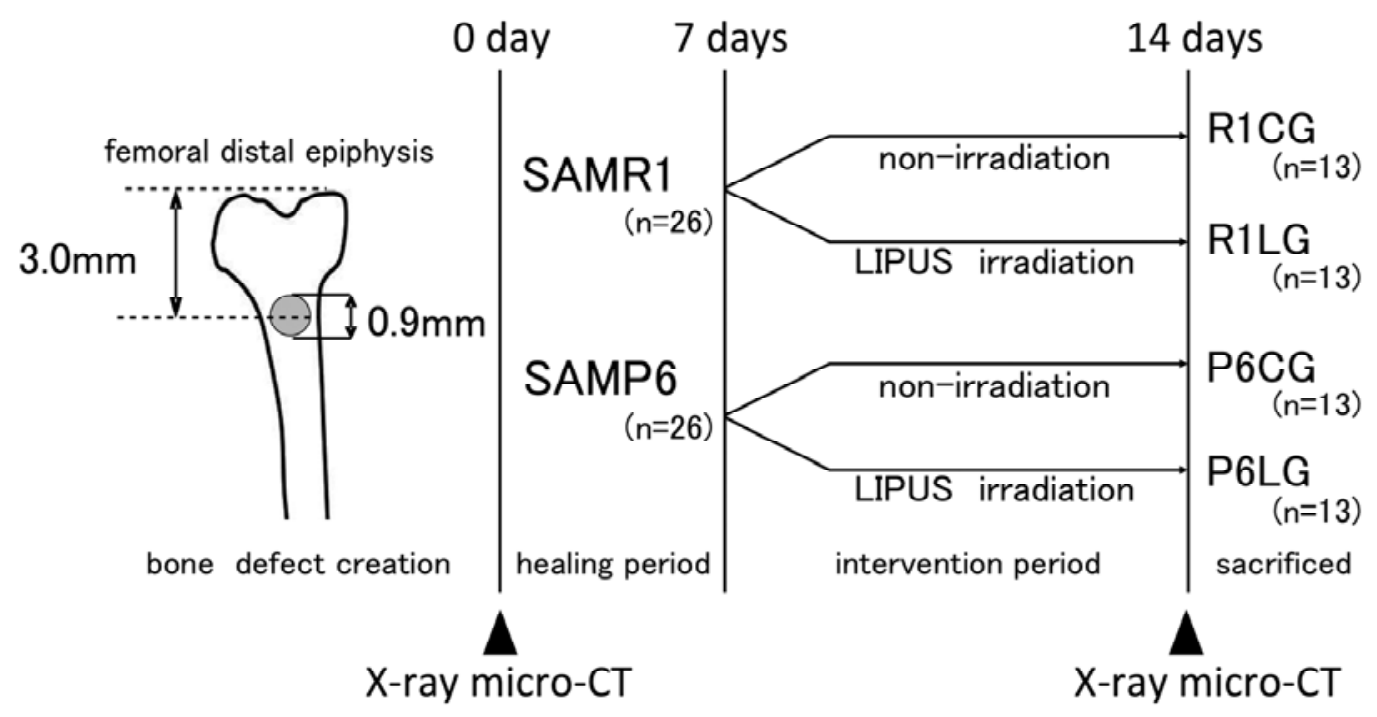

Figure 1. Bone defect model in mouse femur and experimental protocol

of $1-3 \mathrm{MHz}$, in particular, has been reported to accelerate fracture healing in both clinical ${ }^{12,13)}$ and animal studies ${ }^{14,15)}$. Low-intensity pulsed ultrasound was reported to increase expression of osteopontin and osteocalcin, osteoblast differentiation markers, in vitro ${ }^{16-18)}$. In vivo studies have reported that LIPUS irradiation during the fracture healing process increased bone density ${ }^{19)}$, bone mineralization $^{20)}$, and mechanical strength ${ }^{21)}$, and also accelerated bone defect healing ${ }^{22}$. Early-stage osseointegration of implants ${ }^{23)}$ and maintenance of implant stability ${ }^{24)}$ were promoted by LIPUS. Furthermore, LIPUS enhanced healing of fractures under pathological conditions such as diabetes mellitus ${ }^{25)}$ and in nonunion fracture $^{26)}$ and high-turnover osteoporosis (OVX) models ${ }^{27)}$. However, the effect of LIPUS in senile osteoporosis remains to be reported in both sexes. Establishing a means to promote healing and improve the quality of bone in implant patients with lowturnover osteoporosis is an extremely important issue.

The purpose of the present study was to investigate the effects of LIPUS on the healing of bone defects in low-turnover osteoporosis through radiological and histological evaluation.

\section{Materials and Methods}

\section{Surgical bone defect and LIPUS irradiation}

Twenty-week-old male SAMP6 mice (Japan SLC, Inc., Shizuoka, Japan) were used as a model of low-turnover osteoporosis, and 20-week-old male Senescence-Accelerated Mouse Resistant (SAMR1) mice (Japan SLC, Inc., Shizuoka, Japan) as a control. Both groups (each $n=26$ ) were placed under general anesthesia with an intraperitoneal injection of pentobarbital sodium and a $0.9-\mathrm{mm}$ drill-hole bone defect created $3 \mathrm{~mm}$ from the femoral distal epiphysis using a round bur while being careful to avoid damage to the growth plate. Cortical bone perforation was confirmed by bleeding from bone marrow.
At 7 days after bone defect creation, the skin of the surgical defect area was exposed to LIPUS in subgroups of each type of mouse (R1LG: SAMR1 irradiation group; P6LG: SAMP6 irradiation group; $\mathrm{n}=13$ in each group) using the BR Sonic Pro (Ito Co., Ltd., Tokyo, Japan) (diameter of probe: $1.8 \mathrm{~cm}$ ). The following conditions were applied: frequency, $1.0 \mathrm{MHz}$; burstwidth with sine wave, $2000 \mu \mathrm{s}$; repeating pulsation, $100 \mathrm{~Hz}$; and intensity, $320 \mathrm{~mW}$, equivalent to $360 \mathrm{~mW} / \mathrm{cm}^{2}$ spatial average and temporal average (SATA). Irradiation was carried out for $15 \mathrm{~min}$ per day for 6 days. The remaining mice (13 in each group) were defined as non-irradiation groups (R1CG: SAMR1; P6CG: SAMP6). All the mice were sacrificed at 14 days after bone defect creation and the right femur removed after perfusion fixation with $10 \%$ formalin (Wako Pure Chemical Industries, Osaka, Japan) (Fig. 1). All animal experiments were carried out in accordance with the Guidelines for the Treatment of Experimental Animals at Tokyo Dental College (approval number: 223004).

\section{$X$-ray micro-CT and measurement of chronological change}

$\mathrm{X}$-ray micro-CT images were taken with the in vivo 3D micro X-ray CT System R_mCT (Rigaku Corporation, Tokyo, Japan). Micro-CT images were obtained from mouse at immediately after (0-d) and at 14 days after bone defect creation (14-d) under inhalation anesthesia with isoflurane. The samples were set on the object stage and imaging performed over a full 360-degree rotation with an exposure time of $2 \mathrm{~min}$. Conditions were as follows: tube voltage, $85 \mathrm{kV}$; tube current, $160 \mathrm{~mA}$; magnification, $10 \mathrm{x}$; and slice width, $20 \mu \mathrm{m}$. Micro-CT images taken from the same samples at 0 -d and 14-d were merged using the Compare Analysis software (Rigaku Corporation, Tokyo, Japan). Areas of bone that increased over time were indicated in red, those that decreased in blue, and those that showed no change in green in 


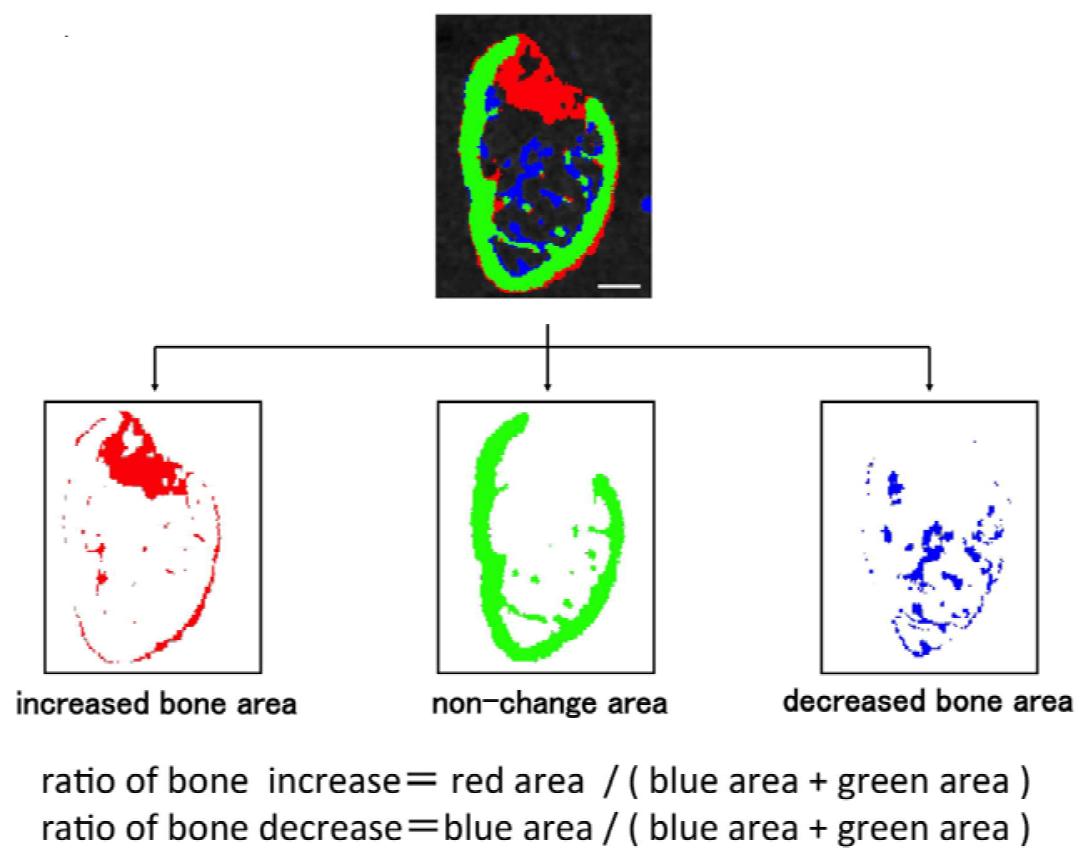

Figure 2. Measurement of chronological change Using Compare Analysis software to analyze chronological change, areas of bone that increased over time were indicated in red, those that decreased in blue, and those where there was no change in green. Bar: $500 \mu \mathrm{m}$

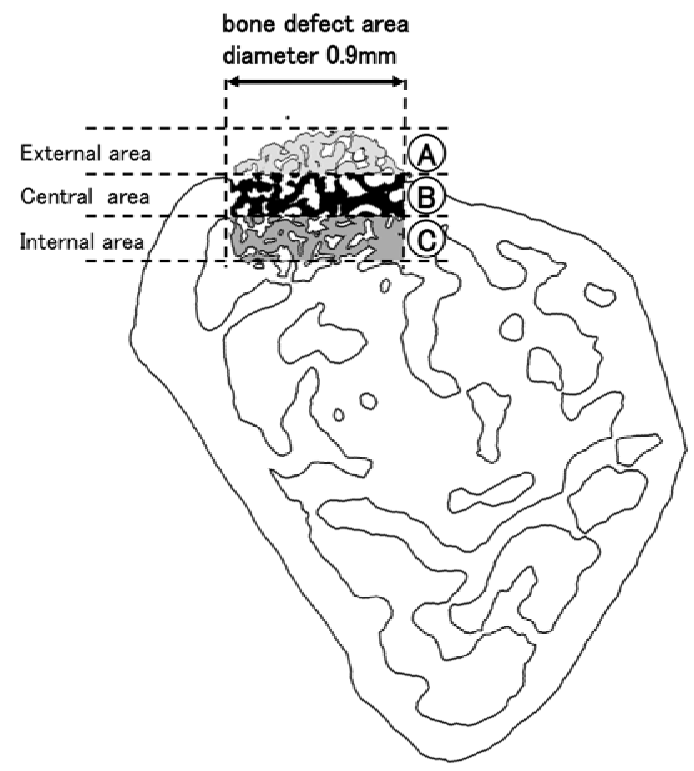

Figure 3. Scheme of area of newly formed bone

New bone in area of bone defect was divided into three areas: central area (B), consisting of rectangle formed by edge of existing cortical bone on either side of bone defect, which corresponded to area of cortical bone damage; external area (A), an area of the same size on outside of defect; and internal area $(\mathrm{C})$, an area of same size on bone marrow.

the merged images. Longitudinal-axis images of 10 sections (200 $\mu \mathrm{m}$ in width) taken from the central point of the bone defect were analyzed by measuring the number of pixels in the increased (red), decreased (blue), and no change areas (green) using Adobe
Photoshop (Adobe Systems, San Jose, USA). Chronological change in bone volume was calculated by comparing change in bone volume between 0 -d and $14-\mathrm{d}$ in the blue and red areas. A further comparison of relative change was then made between the SAMR1 and SAMP6 groups (Fig. 2). The Tukey test was used for the statistical analysis.

\section{Histological analysis and amount of newly formed bone}

The extracted femurs were fixed in $10 \%$ neutral buffered formalin at $4^{\circ} \mathrm{C}$ for 1 day and decalcified with EDTA (pH 7.07.5, Wako Pure Chemical Industries, Osaka, Japan) over 5 days. Paraffin sections of $3 \mu \mathrm{m}$ in thickness were prepared and stained with hematoxylin-eosin (H-E) according to the standard procedure. Histological observation was performed using a universal microscope (Axiophot 2, Carl Zeiss, Oberkochen, Germany). New bone in the bone defect was divided into three areas: a central area, consisting of a rectangle formed by the edge of existing cortical bone on either side of the bone defect, which corresponded to the area of cortical bone damage; an external area, comprising an area the same size on the outside of the defect; and an internal area, comprising an area of the same size in the bone marrow. The ratio of newly formed bone volume was then compared between the irradiation and non-irradiation groups (Fig. 3). In each of these regions of interest, the amount of newly formed bone was measured using Adobe Photoshop. The Mann-Whitney $U$-test was used for the statistical analysis. 

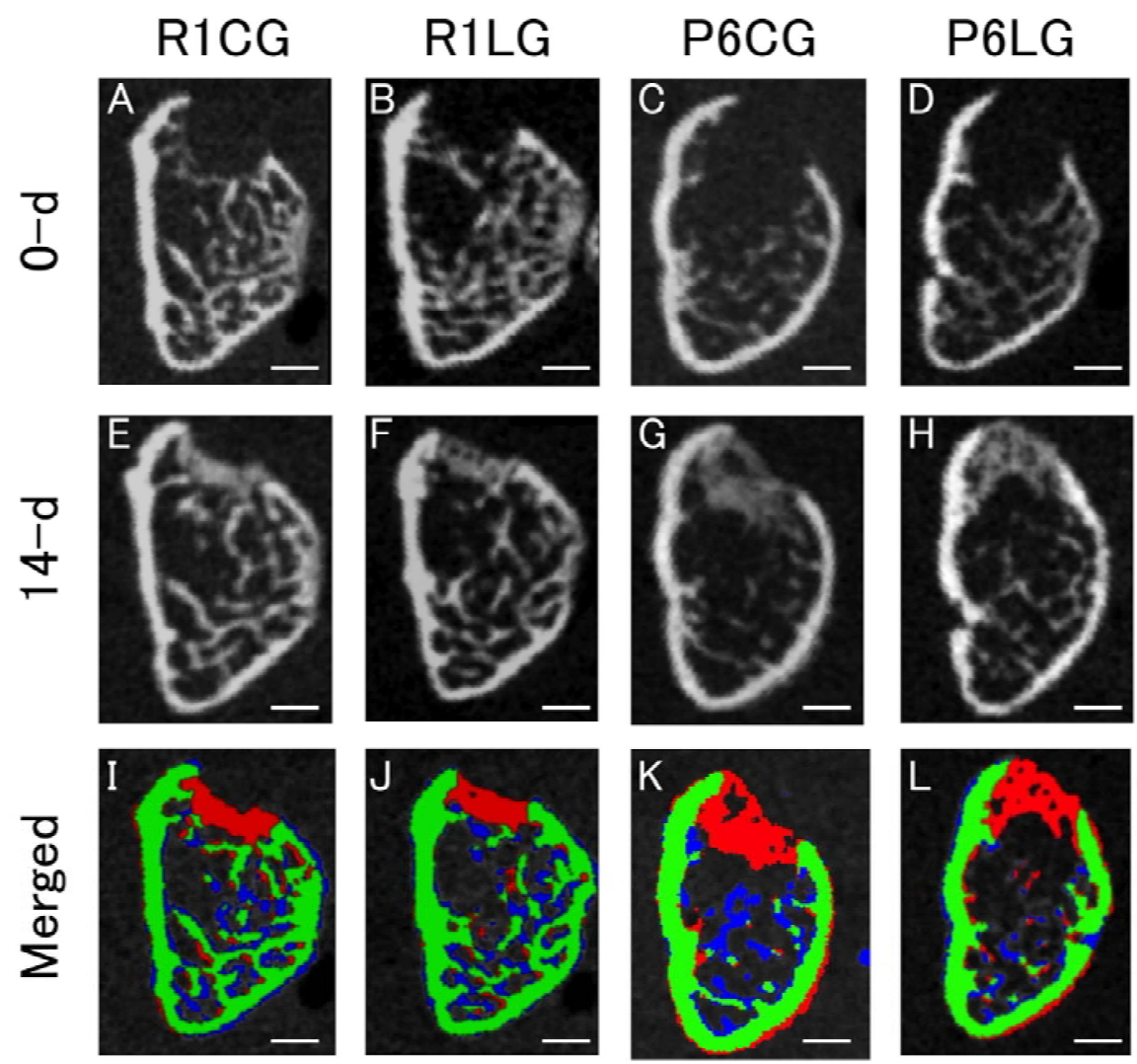

Figure 4. Micro-CT analysis of bone healing at 0 -d and 14-d and merged images At 0-d, cortical bone and trabecular thickness were lower in SAMP6 (P6CG, P6LG) than in SAMR1 (R1CG, R1LG); (A-D). At same time, trabecular callus showing fairly low opacity had formed in defect area in SAMP6 groups (E-H). Blue areas indicating chronological decrease were found mainly in area of trabecular bone, and red area indicating chronological increase was found in cortical bone defect region in both SAMR1 (R1CG, R1LG) and SAMP6 (P6CG, P6LG) (I-L). Bar: $500 \mu \mathrm{m}$
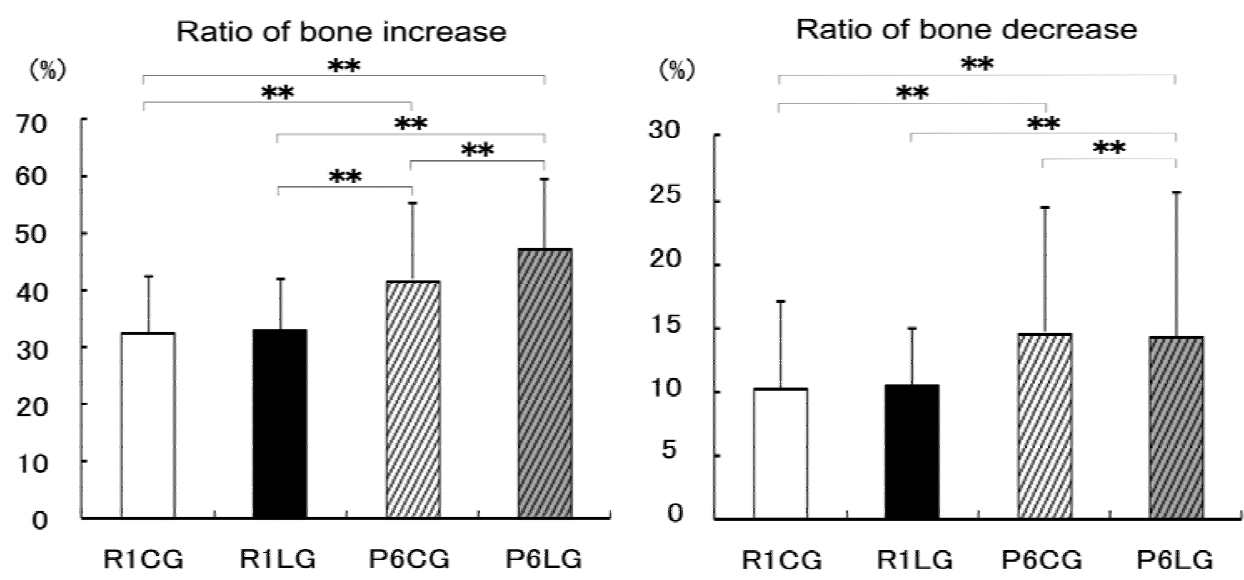

Figure 5. Analysis of increased bone and decreased bone

Ratio of increased bone area between 0-d and 14-d was significantly greater in SAMR1 (R1CG, R1LG) than in SAMP6 (P6CG, P6LG). With ratio of chronological bone decrease, there was significantly greater decrease in SAMR1 than in SAMP6. While no significant difference due to LIPUS irradiation was found between R1CG and R1LG, proportion by which bone increased in P6LG was significantly greater than that in P6CG. And no difference in effect of LIPUS on bone decrease was found between R1CG and R1LG, or between P6CG and P6LG $(* * p<$ 0.01 ; Tukey test).

\section{Immunohistochemical staining}

For immunohistochemical staining, the paraffin sections were deparaffinized with xylene and rehydrated in a series of ethanol.

The sections were washed in $10 \mathrm{nmol} / 1$ phosphate-buffered saline 
R1CG

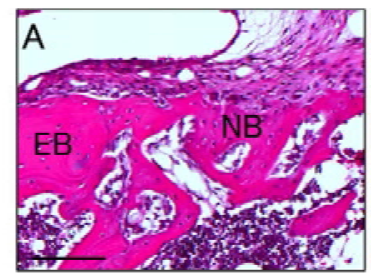

R1LG

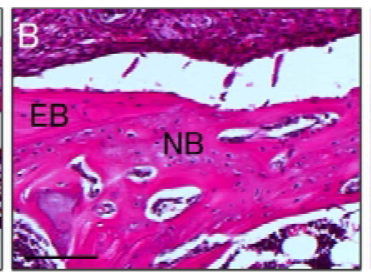

P6CG

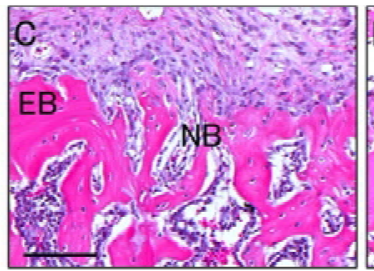

P6LG

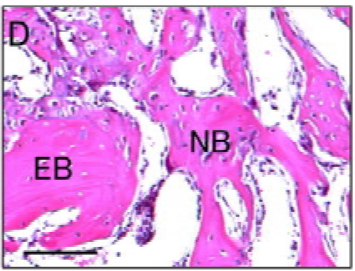

Figure 6. Histological analysis of bone healing process in SAMR1 and SAMP6

Representative micrographs of decalcified H-E stained sections of femur from SAMR1 (A, B) and SAMP6 (C, D) at 14 days after bone defect creation. Woven bone was observed in bone defect area in R1CG (A). Newly formed bone in R1LG showed cortical bone-like structure resembling existing bone (B). In P6CG, immature bone formation was observed originating in existing cortical bone (C). On the other hand, outward new bone formation was observed from periosteum, and newly formed bone formation was observed originating in existing bone, including chondrocytes, in P6LG (D). $\quad \mathrm{EB}=$ existing bone, $\mathrm{NB}=$ new bone, Bar: $100 \mu \mathrm{m}$

(PBS) at a $\mathrm{pH}$ of 7.4 and endogenous peroxidase activity blocked by incubating sections with $0.3 \% \mathrm{H}_{2} \mathrm{O}_{2}$ in methanol for $30 \mathrm{~min}$. After washing in PBS, the sections were reacted with $10 \%$ normal goat serum (Histofine MAX-PO [MULTI]; Nichirei, Tokyo, Japan) for $10 \mathrm{~min}$ to suppress non-specific staining. The sections were then reacted with the primary antibody, rabbit anti-osteocalcin (OC; Enzo Life Sciences, Inc., NY, USA), diluted to 1:500 for 1 hr at below $4^{\circ} \mathrm{C}$. The sections were washed in PBS and then incubated with the secondary antibody, biotinylated anti-mouse IgG antibody (Histofine MAX-PO [MULTI]; Nichirei, Tokyo, Japan), for $1 \mathrm{hr}$ and washed with PBS. The sections were then reacted with peroxidase-labeled streptavidin for $1 \mathrm{hr}$ and washed with PBS. The sections were stained with 3, 3'-diaminobenzidine (DAB) (DAB substrate kit Nichirei, Tokyo, Japan), washed in distilled water, and counterstained with hematoxylin. The sections were then dehydrated according to the established protocol and enclosed sections examined and photographed using a universal microscope.

\section{Results}

\section{Radiological analysis using micro-CT images}

In the micro-CT image obtained at $0-\mathrm{d}$, the width of cortical and trabecular bone in the SAMP6 group (P6CG, P6LG) tended to be thinner compared with that in the SAMR1 group (R1CG, R1LG) (Fig. 4 A-D). At 14-d, a radio-opaque cortical bone-like structure had formed at the bone defect region in the SAMR1 group (Fig. 4 E, F). On the other hand, in the SAMP6 group, a trabecular structure showing fairly low opacity had formed at the defect region (Fig. $4 \mathrm{G}, \mathrm{H}$ ).

In terms of chronological change, merged images obtained at $0-\mathrm{d}$ and 14-d revealed localization of red areas in the cortical bone defect region in both the SAMR1 and SAMP6 groups. On the other hand, blue areas were observed mainly in the area of trabecular bone (Fig. 4 I-L).

The ratio of increased bone area between $0-\mathrm{d}$ and $14-\mathrm{d}$ was significantly greater in the SAMP6 than in the SAMR1 group ( $p$
$<0.01)$. While no significant difference due to LIPUS irradiation was found between R1CG (32.56 $\pm 9.78 \%)$ and R1LG (32.20 \pm $8.94 \%$ ), the proportion by which bone increased in P6LG (47.43 $\pm 12.08 \%)$ was significantly greater than that in P6CG (41.89 \pm $13.54 \%, p<0.01)$. In terms of ratio of decreased bone, a significantly greater decrease was observed in the SAMR1 group (R1CG; $10.26 \pm 6.74 \%$, R1LG; $10.49 \pm 4.56 \%$ ) than in the SAMP6 group (P6CG; $14.65 \pm 9.75 \%$, P6LG; $14.31 \pm 11.30 \%, p$ $<0.01)$. No difference was observed in effect of LIPUS on bone decrease between R1CG and R1LG, or between P6CG and P6LG (Fig. 5).

\section{Histological analysis}

At 7 days after bone defect creation, osteoid formation was recognized in the bone defect area in SAMR1, whereas it was filled with granulation tissue only in SAMP6 (data not shown). At 14-d, woven bone was observed in the bone defect area in R1CG (Fig. 6 A). On the other hand, newly formed bone in R1LG showed a cortical bone-like structure resembling existing bone (Fig. 6 B). New bone in the bone defect area in the SAMP6 groups had a trabecular bone-like structure, and endochondral ossification was observed at the edge of existing bone. In P6CG, immature bone had formed, originating out of the existing cortical bone (Fig. 6 C). On the other hand, new bone in P6LG had formed not only in the bone defect area, but also in the outside area (Fig. 6 D).

\section{Analysis by amount of newly formed bone}

The ratio of new bone growth was greatest in the central area in the SAMR1 group, and that in R1LG was significantly higher than that in R1CG $(p<0.05)$. No significant difference was observed, however, between the internal and external areas (Fig. 7 A). No difference was observed between P6CG and P6LG in the central area in the SAMP6 group. However, in the internal area, the ratio was significantly greater in P6LG $(52.8 \pm 4.4 \%)$ than in P6CG $(44.0 \pm 6.2 \%, p<0.05)$; in the external area, too, 


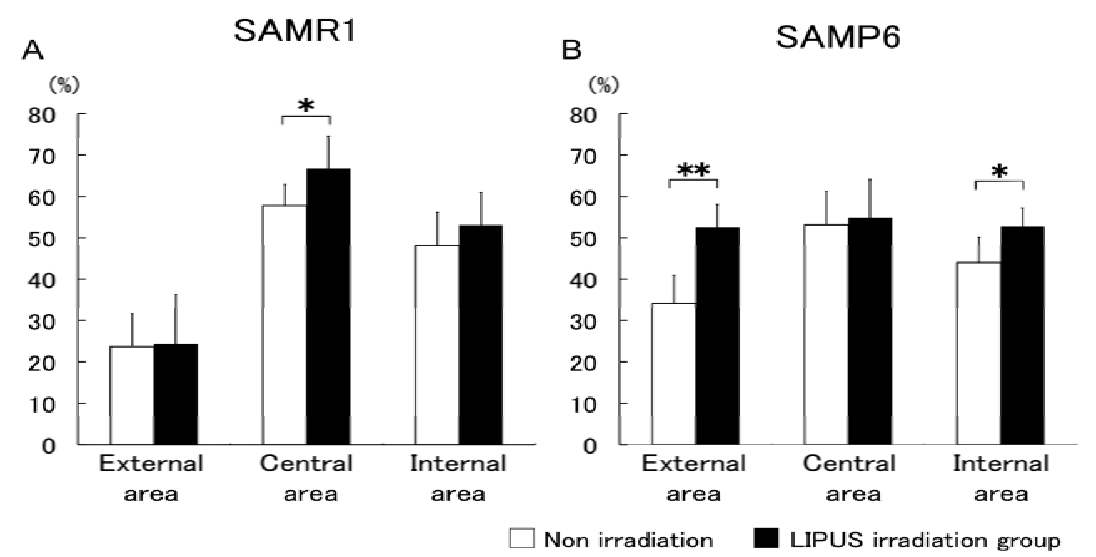

Figure 7. Statistical analysis of amount of newly formed bone in bone defect area at 14 days after bone defect creation

R1LG showed significant increase in newly formed bone in central area compared to R1CG. P6LG showed significant increase in newly formed bone in external area and significant increase in newly formed bone in internal area compared to P6CG $\left({ }^{*} p<0.05,{ }^{* *} p<0.01\right.$; Mann-Whitney U-test $)$.

R1CG

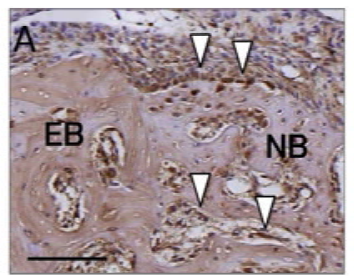

Figure 8. Immunohistochemistry of osteocalcin

Representative micrographs of osteocalcin (OC) distribution in paraffin sections of femur in SAMR1 (A, B) and SAMP6 (C, D, $\mathrm{E}, \mathrm{F})$ at 14 days after bone defect creation. Expression of $\mathrm{OC}$ was found in osteoblasts arranged along new bone filling bone defect in R1CG and on surface of trabecular bone (A). No OC expression was found on surface of new bone in R1LG, but positive reaction was found in osteocytes enclosed within new bone (B). bone, Bar: $100 \mu \mathrm{m}$, Black arrow indicates OC-positive cell

Osteocalcin-positive cells were found localized on surface of newly formed bone originating in outer surface of existing bone in P6CG (C, E). Positive reaction was found among periosteum-derived cells that developed on edge of existing bone and on surface of newly formed bone over whole region from outer to marrow side in P6LG (D, F). EB = existing bone, NB = new the ratio was significantly greater in P6LG $(52.6 \pm 5.5 \%)$ than in P6CG $(34.1 \pm 6.7 \%, p<0.01)$ (Fig. 7 B).

\section{Immunohistochemical staining for osteocalcin}

Expression of osteocalcin was found in osteoblasts in the new bone filling the bone defect in R1CG and on the surface of the trabecular bone (Fig. 8 A). Although no expression of osteocalcin was observed on the surface of either the new or existing cortical bone in R1LG, a slight positive reaction was found in osteoblasts enclosed within the new bone (Fig. 8 B). In P6CG, osteocalcinpositive cells were found localized on the surface of newly formed bone originating from the outer surface of existing bone (Fig. 8 $\mathrm{C}$ ), and a weak positive reaction was recognized on the bone marrow side (Fig. 8 E). However, a positive reaction for osteocalcin was noted among periosteal cells that had developed along the edge of existing bone and on the surface of newly formed bone over the whole region, from the outer side to the bone marrow side, in P6LG (Fig. 8 D, F).

\section{Discussion}

Osteoporosis is a well-known risk factor in implant therapy ${ }^{1,2)}$. Senile osteoporosis, which can develop in either sex, results in a decrease in the thickness of both cortical and trabecular bone due to osteoblastic hypoplasia ${ }^{28)}$. Therefore, from the point of view of dental treatment, it is important to clarify the effect of low-turnover osteoporosis on the process of bone healing.

Many studies have used SAMP6 strain mice in models of lowturnover osteoporosis. Decreased femoral weight and amount of 


\section{Aritomo Yoshida et al.: Effect of Lipus in Senile Osteoporosis}

trabecular bone due to osteoblastic hypoplasia, reduced BMD, bone calcium, and bone phosphorous, and thinning of cortical bone have all been reported in SAMP6 at 16-20 weeks ${ }^{8-10)}$. In the present study, micro-CT images revealed thinner cortical and trabecular bone at 0-d in SAMP6 than in SAMR1. In terms of chronological change, P6CG also showed a significantly greater ratio of decreased bone area than $\mathrm{R} 1 \mathrm{CG}$. This indicates that SAMP6 are suitable for an experimental animal model of senile osteoporosis.

A type of ultrasound stimulation, LIPUS has previously been reported to promote differentiation of osteoblasts in vitro ${ }^{16-18)}$ and healing of fractures in vivo ${ }^{14), 15}$. Moreover, some studies reported that LIPUS affected the healing process in a drill-hole bone defect. Lavendier et al. ${ }^{29}$ investigated the effect of LIPUS at a frequency of $1 \mathrm{MHz}$ and intensities of 100 or $300 \mathrm{~mW} / \mathrm{cm}^{2}$ SATA on a bone defect $3 \mathrm{~mm}$ in diameter in mouse circular calvarial bone, and reported that only $300 \mathrm{~mW} / \mathrm{cm}^{2}$ had an effect. This suggests that the LIPUS conditions used in the present study were appropriate for investigation of its effect on bone defect healing.

Shortinghuis et al. ${ }^{30)}$ reported that a bone defect $5 \mathrm{~mm}$ in diameter in rat mandible was too large for LIPUS to induce bone healing. They suggested that when a bone defect reaches a certain size, the amount of granulation tissue required to form a scaffold for bone regeneration is large, resulting in poor or delayed woundhealing. A micro-CT and histological study of the healing process in a $0.9-\mathrm{mm}$ femoral defect in mice revealed the formation of woven bone at 2 weeks post-operatively, which was remodeled as cortical bone at 4 weeks ${ }^{31)}$. In the present study, similarly woven bone was observed in the bone defect area at 14-d in R1CG. This indicates that the size of the bone defect was appropriate for investigating the healing process. No significant difference was found between R1CG and R1LG in the micro-CT analysis of chronological change. This was probably because chronological measurement was binarized so that differences in bone mineralization would not be reflected, and also because the connective tissue between trabeculae was too small to be examined by micro-CT. Morphological examination revealed that newly formed bone in R1LG was more similar in density to that of existing bone than in R1CG, and that the amount of newly formed bone in the central area was significantly higher in R1LG. Taken together with those of earlier reports, these results indicate that LIPUS enhances bone healing in normally aging mouse.

On the other hand, both micro-CT and morphological examination revealed that new bone had a beam-like structured callus at 14-d in P6CG and that healing was delayed compared with in the SAMR1 group. Although, to our knowledge, no studies to date have evaluated bone defect healing in SAMP6, the healing process of femoral fracture with rigid fixation in SAMP6 has been examined by micro-CT and histologically, with no difference with that in SAMR1 reported ${ }^{32)}$. This result, however, cannot be compared with those obtained in bone defect models, as there is almost no substantial loss of tissue and periosteum-derived cells contribute dominantly to the healing process in fracture models. Yi-Xin He et al. ${ }^{6}$ created a 0.8 -mm diameter drill-hole defect in the femur of OVX mice and compared bone healing at 21 days with that in a non-OVX control group. In the control group, mineralization of the callus in the cortical and bone marrow defect region was found at 7 days after bone defect creation, together with increased mineralization of the callus and partial bridging of the bone defect region at 10 days, and complete ossification of cortical bone at 14 days. In contrast, in the OVX group, there was low mineralization of the callus at 7-10 days after bone defect creation, and a gap with the cortical bone was seen at 14-21 days. The OVX group showed a significantly lower bone volume/tissue volume ratio in the cortical bone region of the bone defect area at 7 days after bone defect creation. At the same time, the OVX group showed significantly lower gene expression of OC, a marker for osteoblastic differentiation, within the callus than the control group. They concluded that the effects on bone healing in the OVX group were the result of interruption of osteoprogenitor cell activity. It was also reported that bone marrow stromal cells in SAMP6 had low alkaline phosphatase (ALP) activity and low mineralization ability. ${ }^{33}$ In the present study, expression of OC in P6CG was localized to the exterior of new bone. This indicates that ossification ability on the bone marrow side was lower in the P6CG than in the SAMR1 group, and that this delayed bone healing.

Bone marrow function was reduced in SAMP6, whereas periosteal derived cell function had no aberrant function compared with SAMR1. Egermann et al. ${ }^{32)}$ found no difference in ALP activity or the mineralization ability of periosteum-derived progenitor cells harvested from femurs between SAMP6 and SAMR1. Silva et al. ${ }^{33)}$ made chronological observations of mineralization in the femur and tibia through calcein labeling. They reported that while endocortical and periosteal mineralization were both observed in SAMR1, only periosteal mineralization was seen in SAMP6. Moreover, they investigated whether mechanical stress by bending affected endocortical and periosteal bone mineral apposition and found no change in the mineralization ability of bone marrow cells. In this study, we used irradiation of a femoral bone defect in SAMP6 by LIPUS as a form of mechanical stress. The analysis of chronological change revealed a significantly higher ratio of bone increase in P6LG than in P6CG. The external and internal areas of new bone volume in P6LG were higher than those in $\mathrm{P} 6 \mathrm{CG}$, while there was no significant difference in the ratio of new bone in the central area. Naruse et al. ${ }^{34)}$ found that irradiation of organ-cultured rat femurs by LIPUS resulted in an increase in OC-positive cells and promotion of differentiation of periosteum-derived stem cells into osteoblasts. Renno et al. ${ }^{35}$ ) irradiated rat tibial bone defects with LIPUS and 
found an increase in OC expression in the irradiated group compared with that in the non-irradiated group during the callus formation period from 7 to 13 days. Ërdogan et al. ${ }^{36}$ irradiated the osteotomized area of rabbit mandible with LIPUS and reported an increase in the thickness in the callus that formed and reduced trabecular separation. In this study, expression of OC was observed in the exterior of new bone in both R1CG and P6CG. This may indicate that the ossification ability of periosteum in SAMP6 was not significantly different from that in SAMR1. These results suggest that bone healing in SAMP6 is delayed in comparison with that in SAMR1 due to reduced differentiation of bone marrow cells into osteogenic cells. Endochondral ossification and outward new bone formation were observed in areas of newly formed bone in SAMP6, indicating that LIPUS promoted proliferation of normal periosteal cells on the surface of the bone and accelerated the differentiation of osteoblasts in the deep region callus. Therefore, a greater amount of new bone was created in the external and internal areas in P6LG than in P6CG. Taken together, these results indicate that LIPUS has the potential to promote new bone formation from cortical bone during tooth extraction and implant placement in patients with senile osteoporosis.

In conclusion, LIPUS has been suggested to accelerate healing on low-turnover osteoporosis by promoting bone formation from periosteum and supplementing reduced bone formation from bone marrow.

\section{Acknowledgments}

The authors would like to thank Associate Professor Jeremy Williams, Tokyo Dental College, for his assistance with the English of this manuscript.

\section{References}

1. Alsaadi G, Quirynen M, Komárek A and van Steenberghe D. Impact of local and systemic factors on the incidence of oral implant failures, up to abutment connection. Clin Periodontol 34: 610-617, 2007

2. August M, Chung K, Chang Y and Glowacki J. Influence of estrogen status on endosseous implant osseointegration. J Oral Maxillofac Surg 59: 1285-1289, 2001

3. Riggs BL, Khosla S and Melton J. A unitary model for involutional osteoporosis: Estrogen deficiency causes both Type I and Type II osteoporosis in postmenopausal women and contributes to bone loss in aging men. J Bone Miner Res 13: 763-773, 1998

4. Duque G and Troen BR. Understanding the mechanisms of senile osteoporosis: New facts for a major geriatric syndrome. J Am Geriatr Soc 56: 935-941, 2008

5. Kalu DN. The ovariectomised rat model of postmenopausal bone loss. Bone Miner 15: 175-191, 1991

6. He YX, Zhang G, Pan XH, Liu Z, Zheng LZ, Chan CW, Lee
KM, Cao YP, Li G, Wei L, Hung LK, Leung KS and Qin L. Impaired bone healing pattern in mice with ovariectomyinduced osteoporosis: A drill-hole defect model. Bone 48: 1388-1400, 2011

7. Duarte PM, César Neto JB, Gonçalves PF, Sallum EA and Nociti FH. Estrogen deficiency affects bone healing around titanium implants: a histometric study in rats. Implant Dent 12: 340-346, 2003

8. Matsushita M, Tsuboyama T, Kasai R, Okumura H, Yamamuro T, Higuchi K, Higuchi K, Kohno A, Yonezu T and Utani A. Age-related changes in bone mass in the senescenceaccelerated mouse (SAM). SAM-R/3 and SAM-P/6 as new murine models for senile osteoporosis. Am J Pathol 125: 276-283, 1986

9. Kasai S, Shimizu M, Matsumura T, Okudaira S, Matsushita $\mathrm{M}$, Tsuboyama $\mathrm{T}$, Nakamura $\mathrm{T}$ and Hosokawa $\mathrm{M}$. Consistency of low bone density across bone sites in SAMP6 laboratory mice. J Bone Miner Metab 22: 207-214, 2004

10. Chen $H$, Shoumura $S$ and Emura S. Ultrastructural changes in bones of the senescence-accelerated mouse (SAMP6): a murine model for senile osteoporosis. Histol Histopathol 19: 677-685, 2004

11. Rubin C, Bolander M, Ryaby JP and Hadjiargyrou M. The use of low-intensity ultrasound to accelerate the healing of fractures. J Bone Joint Surg Am 83: 259-270 2001

12. Kristiansen TK, Ryaby JP, McCabe J, Frey JJ and Roe LR. Accelerated healing of distal radial fractures with the use of specific, low-intensity ultrasound. A multicenter, prospective, randomized, double-blind, placebo-controlled study. J Bone Joint Surg Am 79: 961-973, 1997

13. Heckman JD, Ryaby JP, McCabe J, Frey JJ and Kilcoyne RF. Acceleration of tibial fracture-healing by non-invasive, lowintensity pulsed ultrasound. J Bone Joint Surg Am 76: 2634, 1994

14. Duarte LR. The stimulation of bone growth by ultrasound. Arch Orthop Trauma Surg 101: 153-159, 1983

15. Dyson $M$ and Brookes $M$. Stimulation of bone repair by ultrasound. Ultrasound Med Biol 2: 61-66, 1983

16. Sena K, Leven RM, Mazhar K, Sumner DR and Virdi AS. Early gene response to low-intensity pulsed ultrasound in rat osteoblastic cells. Ultrasound Med Biol 31: 703-708, 2005

17. Warden SJ, Favaloro JM, Bennell KL, McMeeken JM, Ng KW, Zajac JD and Wark JD. Low-intensity pulsed ultrasound stimulates a bone-forming response in UMR-106 cells. Biochem Biophys Res Commun 286: 443-450, 2001

18. Sant'Anna EF, Leven RM, Virdi AS and Sumner DR. Effect of low intensity pulsed ultrasound and BMP-2 on rat bone marrow stromal cell gene expression. J Orthop Res 23: 646652,2005 


\section{Aritomo Yoshida et al.: Effect of Lipus in Senile Osteoporosis}

19. Mayr E, Laule A, Suger G, Rüter A and Claes L. Radiographic results of callus distraction aided by pulsed low-intensity ultrasound. J Orthop Trauma 15: 407-414, 2001

20. Iwai T, Harada Y, Imura K, Iwabuchi S, Murai J, Hiramatsu K, Myoui A, Yoshikawa H and Tsumaki N. Low-intensity pulsed ultrasound increases bone ingrowth into porous hydroxyapatite ceramic. J Bone Miner Metab 25: 392-399, 2007

21. Wang SJ, Lewallen DG, Bolander ME, Chao EY, Ilstrup DM and Greenleaf JF. Low intensity ultrasound treatment increases strength in a rat femoral fracture model. J Orthop Res 12: 40-47, 1994

22. Hasuike A, Sato S, Udagawa A, Ando K, Arai Y and Ito K. In vivo bone regenerative effect of low-intensity pulsed ultrasound in rat calvarial defects. Oral Surg Oral Med Oral Pathol Oral Radiol Endod 111: 12-20, 2011

23. Ustun Y, Erdogan O, Kurkcu M, Akova T and Damlar I. Effects of low-intensity pulsed ultrasound on dental implant osseointegration: A preliminary report. Eur J Dent 2: 254262, 2008

24. Hsu SK, Huang WT, Liu BS, Li SM, Chen HT and Chang CJ. Effects of near-field ultrasound stimulation on new bone formation and osseointegration of dental titanium implants in vitro and in vivo. Ultrasound Med Biol 37: 403-416, 2011

25. Gebauer GP, Lin SS, Beam HA, Vieira P and Parsons JR. Low-intensity pulsed ultrasound increases the fracture callus strength in diabetic BB Wistar rats but does not affect cellular proliferation. J Orthop Res 20: 587-592, 2002

26. Takikawa S, Matsui N, Kokubu T, Tsunoda M, Fujioka H, Mizuno K and Azuma Y. Low-intensity pulsed ultrasound initiates bone healing in rat nonunion fracture model. J Ultrasound Med 20: 197-205, 2001

27. Cheung WH, Chin WC, Qin L and Leung KS. Low intensity pulsed ultrasound enhances fracture healing in both ovariectomy-induced osteoporotic and age-matched normal bones. J Orthop Res 30: 129-136, 2012

28. Parfitt AM, Villanueva AR, Foldes J and Rao DS. Relations between histologic indices of bone formation: Implications for the pathogenesis of spinal osteoporosis. J Bone Miner Res 10: 466-473, 1995

29. Lavandier B, Gleizal A and Béra JC. Experimental assessment of calvarial bone defect re-ossification stimulation using lowintensity pulsed ultrasound. Ultrasound Med Biol 35: 585594, 2009

30. Schortinghuis J, Ruben JL, Raghoebar GM and Stegenga B. Ultrasound to stimulate mandibular bone defect healing: A placebo-controlled single-blind study in rats. J Oral Maxillofac Surg 62: 194-201, 2004

31. Monfoulet L, Rabier B, Chassande O and Fricain JC. Drilled hole defects in mouse femur as models of intramembranous cortical and cancellous bone regeneration. Calcif Tissue Int 86: 72-81, 2010

32. Egermann M, Heil P, Tami A, Ito K, Janicki P, Von Rechenberg B, Hofstetter W and Richards PJ. Influence of defective bone marrow osteogenesis on fracture repair in an experimental model of senile osteoporosis. J Orthop Res 28: 798-804, 2010

33. Silva MJ, Brodt MD, Ko M and Abu-Amer Y. Impaired marrow osteogenesis is associated with reduced endocortical bone formation but does not impair periosteal bone formation in long bones of SAMP6 mice. J Bone Miner Res 20: 419427, 2005

34. Naruse K, Mikuni-Takagaki Y, Urabe K, Uchida K and Itoman M. Therapeutic ultrasound induces periosteal ossification without apparent changes in cartilage. Connect Tissue Res 50: 55-63, 2009

35. Renno AC, Fávaro-Pípi E, Fernandes K, Tim C and Ribeiro DA. Ultrasound therapy modulates osteocalcin expression during bone repair in rats. Ultrasonics 52: 111-116, 2012

36. Ërdogan Ö, Esen E, Ustün Y, Kürkçü M, Akova T, Gönlüen G, Uysal H and Cevlik F. Effects of low-intensity pulsed ultrasound on healing of mandibular fractures: an experimental study in rabbits. J Oral Maxillofac Surg 64: 180-188, 2006 
J.Hard Tissue Biology Vol. 22(3):301-310, 2013 